Recepción: 13 / 05 / 2018

Aceptación: 29 / 07 / 2018

Publicación: 05 / 10 / 2018

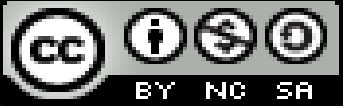

Ciencias de la educación

Artículo de investigación

\title{
El desarrollo del aprendizaje a nivel superior mediante plataformas Mooc
}

\author{
The development of learning at a higher level through Mooc platforms
}

O desenvolvimento da aprendizagem a um nível mais alto pelas plataformas

\section{Mooc}

\author{
César V. Ramírez-Gutiérrez ${ }^{\mathrm{I}}$ \\ cesar.ramirezg@ug.edu.ec \\ Jacqueline A. Villacís-Tagle II \\ jaalvill@espol.edu.ec \\ Lissenia I. Sornoza-Quijije ${ }^{\text {III }}$ \\ 1sornoza@espol.edu.ec
}

Correspondencia: cesar.ramirezg@ug.edu.ec

\footnotetext{
${ }^{I}$ Magister en Educación Informática, Licenciado en Ciencias de la Educación Mención Informática y Programación, Docente Universidad de Guayaquil, Guayaquil, Ecuador.

${ }^{\text {II }}$ Master Universitario en Postproducción Digital, Licenciada en Diseño y Producción Audiovisual, Escuela Superior Politécnica del Litoral, Guayaquil, Ecuador.

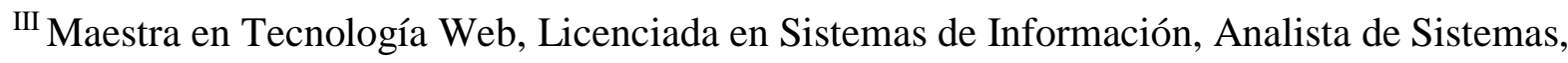
Escuela Superior Politécnica del Litoral, Guayaquil, Ecuador.
} 


\section{Resumen}

Este trabajo refleja el estudio de autores realizado sobre una de las herramientas favorables para el aprendizaje a distancia que desde hace mucho tiempo se ha estado utilizado en países anglosajones y de la Unión Europea, por su alto impacto en la educación superior y que en Ecuador muy poco se ha hablado y utilizado en función de tomar el mayor provecho de su utilidad. Estas son las plataformas MOOC (Cursos Abiertos Online y Masivos).

Se ha considerado el análisis de los MOOC, por las ventajas que se obtiene en el uso de esta herramienta tecnológica, ya que permite el manejo de una variada colección de recursos multimedia, bibliográficos y contenidos de temas específicos en línea y de libre acceso.

El objetivo principal de este trabajo es impulsar la aplicación de esta herramienta en la educación superior para la participación activa y colaborativa entre tutores y estudiantes. El aprendizaje colaborativo que se desarrollan en estas plataformas consolidan la aplicación de una metodología Andragógica y de autoaprendizaje entre los participantes.

Se puede concluir que es importante la implementación de estos cursos en las universidades del país, ya que favorece a los participantes con la interacción de contenidos confiables y veraces, esto permitirá la expansión de forma colectiva a otras instituciones de otros países.

Palabras clave: MOOC; COMA; e-learning; tecnología de aprendizaje; Cursos Abiertos Online y Masivos.

\section{Abstract}

This work reflects the study of authors carried out on one of the favorable tools for distance learning that has been used for a long time in Anglo-Saxon countries and the European Union, due to its high impact in higher education and in Ecuador. Little has been spoken and used to take advantage of its usefulness. These are MOOC platforms (Open Online and Massive Courses).

The analysis of MOOCs has been considered, due to the advantages obtained in the use of this technological tool, since it allows the management of a varied collection of multimedia, bibliographic and content resources of specific online and freely accessible subjects.

The main objective of this work is to promote the application of this tool in higher education for active and collaborative participation between tutors and students. The collaborative learning developed in these platforms consolidates the application of an andragogical methodology and 
It can be concluded that it is important to implement these courses in the universities of the country, since it favors the participants with the interaction of reliable and truthful content, this will allow the expansion of collective form to other institutions in other countries.

Key words: MOOC; COMA; e-learning; learning technology; Massive Open Online Courses.

\section{Resumo}

Este trabalho reflete o estudo de autores realizado sobre uma das ferramentas favoráveis ao ensino a distância que vem sendo utilizada há muito tempo em países anglo-saxônicos e na União Européia, devido ao seu alto impacto no ensino superior e no Equador. Pouco tem sido falado e usado para tirar proveito de sua utilidade. Estas são plataformas MOOC (Open Online e Massive Courses).

A análise dos MOOCs tem sido considerada, devido às vantagens obtidas no uso desta ferramenta tecnológica, uma vez que permite o gerenciamento de uma coleção variada de recursos multimídia, bibliográficos e de conteúdo, de assuntos on-line específicos e de livre acesso.

O principal objetivo deste trabalho é promover a aplicação desta ferramenta no ensino superior para a participação ativa e colaborativa entre tutores e alunos. A aprendizagem colaborativa desenvolvida nessas plataformas consolida a aplicação de uma metodologia andragógica e a autoaprendizagem entre os participantes.

Pode-se concluir que é importante implementar esses cursos nas universidades do país, pois favorece aos participantes a interação de conteúdo confiável e verdadeiro, o que permitirá a ampliação da forma coletiva para outras instituições de outros países.

Palavras chave: MOOC; COMA; e-learning; tecnologia de aprendizagem; Cursos Abertos Online e Massivo.

\section{Introducción}

Tal como lo indican Vázquez-Cano, E., \& Meneses, E. L. (2015), los MOOC en este panorama de la educación en abierto y gratuita surgen como una necesidad de especialización que no conlleve una acreditación o certificación como objetivo prioritario pero que favorezca un 
acercamiento a nuevas realidades laborales y científicas que las propuestas de enseñanzas regladas más encorsetadas no pueden ofrecer. Por ello, los MOOC han acaparado este interés mundial debido a su gran potencial para ofrecer una formación gratuita y accesible a cualquier persona independientemente de su país de procedencia, su formación previa y sin la necesidad de pagar por su matrícula

Siguiendo el enfoque en el que ven los MOOC, Mancera, D. T., \& Saldaña, D. G. (2014) analizan que los MOOC se sustentan de contenidos libres y flexibles de páginas web, wikis, recursos educativos abiertos y redes sociales, y pueden escalarse para ajustarse a la demanda. Es un aprendizaje en el que los conocimientos se adquieren de la comunidad y de los sujetos que trabajan juntos (aprendizaje social y peer to peer). Las actividades propuestas siguen una metodología basada en retos y juegos, con objeto de que los participantes puedan participar de forma práctica y lúdica como parte motivacional del aprendizaje.

El desarrollo de la tecnología se ha convertido en una variable de contantes cambios y desarrollos avanzado, abarcando en su mayoría todas las áreas de conocimiento para poder dar solución a diversos problemas o para la satisfacción de necesidades que antes se veían complejas e inalcanzables.

En este escenario al hablar del como la tecnología ha favorecido en gran escala el área de la educación logrando la transformación de paradigmas y nuevos modelos y métodos de enseñanza aprendizaje a nivel de educación básica, bachillerato y superior, permitiendo así proyectar soluciones prácticas para disminuir la brecha de alfabetismo y generar conocimiento global, horizontal, participativo e hipertextual.

Analizando este campo globalizado donde las fronteras desaparecen gracias a la aplicación de las nuevas tecnologías de información y comunicación, la educación adquiera nuevos retos, compromisos y roles en los cual la visión del proceso de enseñanza aprendizaje amplía su perspectiva para poder dirigir el conocimiento ya que con la utilización de los recursos tecnológicos el aula de clase deja de ser un lugar de cuatro paredes una pizarra. 
El buen aprovechamiento del uso de la Internet en ámbitos de la educación según Aretio, L. G. (2015), nos permite llevar información práctica y de debate a plataformas virtuales con fines de enseñanza y aprendizaje colectivo. Es por esto que podemos decir que el conocimiento en la actualidad lo podemos construir desde cualquier parte del mundo, los retos de la educación involucran el diseño de currículos locales, concepto que integra lo local desde un perspectiva y proyección global, además estos currículos involucran la producción de recursos hipermediales y la oferta de cursos masivos entre otros, una de esas nuevas soluciones son los MOOC.

\section{Importancia del Problema}

La formación universitaria es una activada creciente en demanda y costos las TICS han permitidos involucrarnos en cambios fundamentales como la competencia global, accesos educativos abiertos y nuevas metodologías didácticas derivadas de las interacciones sociales y el aprendizaje colaborativo.

Conforme lo indica Caldera-Serrano, J., \& León-Moreno, J. (2015), los MOOC están considerados de forma positiva en la comunidad universitaria norteamericana, esto incluye a las universidades de gran prestigio, las mismas que de forma presencial reflejan un número de estudiantes considerable debido a su amplio prestigio y bien posicionado en rankings internacionales. Entonces cabe preguntarse el porqué de ofertar formación MOOC's. Que Harvard, Stanford, o el Massachusetts Institute of Technology (MIT) ofrezcan sus propios cursos mediante la modalidad MOOC puede ser visto como una forma de hacerse competencia a sí mismos, una forma de ampliar su catálogo formativo, o de perjudicarse a sí mismos por crear una "línea blanca" de la educación.

Modelo de enseñanza aprendizaje permiten hacer el seguimiento de los participantes sin precedente ya que podemos saber cuándo se conectan, que tiempo han estado trabajando en la plataforma, la utilización de los materiales utilizados para el curso como foros, cuestionarios, descarga de archivos o reproducción de multimedia para poder realizar las actividades establecidas como tareas del tema en el cual están cursando.

La idealización de los Cursos Abiertos Online Masivos (MOOC) nacieron en base al desarrollo de las tecnologías y al ritmo en que la información cada día es compartida con mayor rapidez y de fácil acceso, más aún si este contenido es de carácter formativo, muchos usuarios interesados como docentes, estudiantes o instituciones de desarrollo de conocimiento forma parte de esta 
herramienta que permite el compartir y participar de forma colaborativa de nuevas ideas y experiencias de todas partes del mundo.

Según Del Cura, M. (2013) señala que los MOOCs están alineados según la teoría del conectivismo y la pedagogía abierta, basada en el aprendizaje en red. El MOOC como ya lo habíamos descrito es un curso, es abierto, es participativo, es distribuido por ende esto permite y ratifica él apoya al aprendizaje online en redes.

\section{Metodología}

Tal como indica Poy, R., \& Gonzales-Aguilar, A. (2014) que el diseño pedagógico de las plataformas MOOC, es sin duda uno de los elementos más relevantes de cara a optimizar los resultados del aprendizaje de los usuarios de estas plataformas, dado que para que sea posible la puesta en marcha de los procesos educativos, es necesario que se tenga en cuenta, en primer lugar, la fundamentación teórica del modelo pedagógico que los sustenta a fin de determinar las necesidades pedagógicas a las que se les ha de dar respuesta cuando se procede al diseño pedagógico de los entornos virtuales de aprendizaje, cuyo rasgo propio es que el interfaz entre el sujeto que aprende y el diseñador de la práctica educativa es la tecnología (García, 2002).

Tanto las plataformas MOOC como otros productos e-learning denominados como software educativo 3.0. y que incluye, por ejemplo, los denominados juegos serios (serious games) procedentes del Digital Gamebased Learning, responden a la necesidad de emplear metodologías innovadoras para la consecución de una formación de impacto en el ámbito de los procesos de enseñanza aprendizaje en entornos virtuales de aprendizaje, principalmente en cuanto al grado de participación activa proporcionado al sujeto del aprendizaje (Prensky, 2010).

Precisamente los entornos virtuales de aprendizaje tienen cada vez mayor presencia en el conjunto de la oferta formativa, pudiendo afirmar que, en general, la población que utiliza los actuales canales de aprendizaje on line manifiesta nuevas tendencias como el hecho de tener una mayor demanda de contenidos innovadores respecto a los más tradicionales modelos de aprendizaje propios de la enseñanza presencial (Krichen, 2007). En consecuencia, en ambos casos, las plataformas MOOC responden a esa nueva demanda de contenidos innovadores para las nuevas generaciones de usuarios crecientemente inmersos en la sociedad del conocimiento y de la información. 
Con el fin de analizar desde el punto de vista del diseño pedagógico, la construcción de las diferentes plataformas MOOC, hemos utilizado el modelo de Freitas y Jarvis (2007) que toma en cuenta tres elementos de análisis más la representación: el contexto, el aprendiz, y la pedagogía usada, además de la propia representación digital del producto e-learning, como cuarto elemento adicional.

Las plataformas MOOC se fundamentan mayoritariamente en un modelo pedagógico socio cognitivo, donde los estudiantes construyen significados por sí mismos, como lo ponen de manifiesto la presencia de las variables que caracterizan este modelo teórico.

Sin embargo, Poy, R., \& Gonzales-Aguilar, A. nos dice que han observado cómo las plataformas MOOC tienen elementos del diseño pedagógico asentado en los modelos conductistas, como lo demuestran el hecho de aquellas plataformas que contienen información para ser transmitida mediante el entrenamiento en las tareas a través de ejercicios, en donde el paso al siguiente nivel de dificultad viene determinado de antemano.

Otras propuestas metodológicas que encontramos en las plataformas MOOC se circunscriben dentro de los fundamentos teóricos del aprendizaje situado, en donde el contexto adquiere un papel esencial (Brown et al., 1989). Este enfoque es muy importante y se encuentra en la base de algunas de las propuestas metodológicas de las plataformas MOOC, en las que a partir del planteamiento de una situación compleja y problematizada y a través de la reflexión conjunta se van proponiendo nuevas tareas y retos a resolver por los participantes de la plataforma, de modo que se simulan o representan figuradamente los supuestos aplicables a un entorno real.

\section{Características de los MOOC's}

Ya se ha indicado cual es la definición de MOOC de la misma forma cómo surgió y como alcanzo la popularidad que en la actualizar represente en distintas universidades de gran prestigio, pues es hora de indicar cuales son las características que los MOOC cumplen para el desarrollo de un curso:

\section{Es un Curso}

Un curso según su característica cuenta personas con un conocimiento específico tiene el rol de facilitadores o capacitadores, existen también los recursos que serán de utilidad para dicha capacitación y apoyo para los estudiantes. Se debe tener en cuente que al referirnos de un MOOC 
no estamos indicando que es una institución educativa estatal o particular, ni tampoco es la realización de un solo modulo sino todo lo contrario, es la participación colaborativa de todos los integrantes que se han registrado para poder así aumentar su conocimiento en un tema específico de interés y desarrollo constructivista.

\section{Es Abierto}

Es abierto por la acción que se les permite realizar a los usuarios participantes del MOOC esto quiere decir a la accesibilidad de la información ya sea de forma visual, descargable y de participación en foros o debates de discusión y opinión. Con esto también cuando hablamos de abierto se debe de considerar que el acceso no tengo cuota de ingreso o de inscripción, ósea que no tenga costo económico alguno para ser parte del MOOC como estudiante.

\section{Es en Línea}

Es En línea ya que gracias el desarrollo acelerado del Internet ha permitido creación de plataformas de comunicación en tiempo real para estar cada vez más cerca de contenido que antes era difícil obtener. En la actualidad desde nuestros teléfonos inteligentes podemos tener acceso de información generada al momento de los hechos, así como también compartirla a tan solo un clic. Considerando lo dicho por González, H. M., \& Ávila, A. P. (2014) las webs 2.0 permiten la interacción más eficaz y fácil con paradas con la web anterior, esto ha sido la herramienta tecnológica que los estudiantes utilizan como recurso diario para relacionarse con otros estudiantes de su misma institución o de otras.

Os blogs, videos blogs, cuentas de redes sociales entre otros son los componentes de comunicación en los que diariamente las personas utilizamos para esta al día con lo que pasa a nuestro alrededor, más aún nos ayudan a ser partícipes de programas de aprendizajes no solo de manera local sino también a nivel mundial. Las brechas que existían entre la enseñanza y el aprendizaje, cada vez se acortan y nos aproximan día a día.

\section{Es Masivo}

Es Masivo los MOOC según lo indican Castaño, C., Maiz, I., \& Garay, U. (2015) ya que presentan una nueva manera de enfocar la formación que está atrayendo a millones de participante de todas partes del mundo, y que está alternando la manera en que las universidades 
presenciales programen la formación en modo online. Los MOOC se han transformado en la evolución del e-learning.

Para algunos analistas, las herramientas Web 2.0 que subyacen a estas nuevas formas de elearning ocasionan una transformación del espacio social al ofrecer a los individuos nuevas oportunidades de aprendizaje rompiendo las barreras tradicionales del espacio y el tiempo de la formación reglada en el modelo global, conforme comentan en su artículo Poy, R., \& GonzalesAguilar, A. (2014).

Tal como lo indican Caldera-Serrano, J., \& León-Moreno, J. (2015), los MOOCs han creado una conversión positiva de alto impacto en la educación universitaria, ya que son un claro ejemplo de formación a distancia planificados para alcanzar un número ilimitado de usuarios, debido a su carácter abierto, participativo y con una metodología basada en el conocimiento.

Los MOOCs han tenido la aceptación en ciertas instituciones educativas a nivel superior en el ecuador, aunque otras universidades aún no han implementado este sistema de cursos masivos abiertos, esto poder deberse al desconocimiento del uso de esta herramienta o por falta de personal capacitado para la aplicación de esta plataforma, privándose de las grandes ventajas y opciones que nos brinda esta tecnología aplicada en la web 2.0

Considerando que una de las mejores universidades como la es Harvard, Stanford, y el Massachusetts Institute of Technology (MIT) han participado de esta herramienta tecnología online con sus cursos mediante los MOOCs por su efectividad y fácil acceso para estudiantes de todas partes del mundo, y por este medio poder expandir la oportunidad de distribución de conocimiento a sus participantes que aspiran un crecimiento académico de tan prestigiosas instituciones educativas es estatus superior.

\section{CLASIFICACIÓN DE LOS MOOC}

cMOOC: Enfoque conectivista

Basados en aprendizaje conectivista centrados en el conocimiento que se genera en la participación y contribución de los estudiantes más que en el contenido presentado. Tal como indican Bartolomé, A., \& Steffens, K. (2015), los cMOOC se fortalecen el conocimiento distribuido en la red la función del tutor es mínima o nula y las evaluaciones se las llevan 
generalmente de forma automatizada o son los propios estudiantes quienes evalúan su autoaprendizaje al final del módulo. Este tipo de MOOC es ideal para cursos donde el estudiante busca un refuerzo a un tema específico.

xMOOC: Con un enfoque basado en el conductivismo

Este tipo de Mooc están basados en el aprendizaje tipo conductista centrados en el conocimiento que se genera principalmente por medio de videos de corta duración que son realizados por un tutor o por un experto, quien también tiene participación constante durante el desarrollo del curso y da seguimiento a los participantes. Los xMOOC conforme Blanco, Á. et al. (2014) se fortalecen con la interacción que se genera por los diferentes canales de comunicación a los que se pueden agregar las redes sociales que contribuyen en minimizar la individualización del estudiante, por la cantidad de participantes de los xMOOC la revisión personalizada de asignaciones por parte del tutor se hace imposible por lo que se emplean a más de evaluaciones automatizadas el sistema de revisión peer to peer, donde los estudiantes tienen la oportunidad de revisar y retroalimentar las tareas de sus compañeros.

Los cursos basados en modelo xMOOC consisten básicamente en una selección de videos con el complemento de un chat o foro de discusión y opinión, muchos profesores e investigadores consideran que el modelo de enseñanza en los MOOCs no es diferente a las plataformas de canales videos blogs.

\section{bMOOC: Modelo hibrido}

Es una nueva y más completa de los modalidad de los MOOCs, originada con la intención de minimizar la deserción de los estudiantes participantes al buscar mejores alternativas de participación y socialización, están basado en el aprendizaje cognotivista, centrados en la interacción humana el conocimiento lo generan a través de presentación de videos instruccionales que pueden ser comentados, lecturas y sesiones cara a cara con el tutor del curso, el tutor también tiene participación constante durante el desarrollo del curso explicando y promoviendo la participación.

Los bMOOC se fortalecen con la interacción que tienen los estudiantes en discusiones sincrónicas, Mancera, D. T., \& Saldaña, D. G. (2014) indican que además de implementar 
modelos de evaluación automatizada peer to peer también utilizan evaluación grupal, los cursos donde se desean compartir técnicas o nuevas metodologías pueden utilizar este tipo de MOOC.

SMOOC: Dirigidos a grupos más pequeños

Estos MOOC comparados con los otros tipos de cursos, agrupan pequeñas comunidades de usuarios esto permite concentrarse en las características individuales del estudiante y potenciar la interacción face to face en términos de intimidad, confianza, apoyo y seguridad. Los sMOOC permiten a novatos y expertos interactuar libremente.

\begin{tabular}{|c|c|c|c|}
\hline \multicolumn{4}{|c|}{ TABLA DESCRIPTIVA DE LOS TIPOS DE MOOC } \\
\hline $\begin{array}{l}\text { TIPOS DE } \\
\text { MOOC }\end{array}$ & CONTENIDOS & COMUNICACIÓN & EVALUACIÓN \\
\hline $\begin{array}{l}\text { cMOOC } \\
\text { Modelo conectivista } \\
\text { basado en redes de } \\
\text { comunicación }\end{array}$ & $\begin{array}{l}\text { - Lecturas } \\
\text { - Videos disponibles en la } \\
\text { red } \\
\text { - Recursos educativos }\end{array}$ & $\begin{array}{l}\text { - Dentro o fuera de la } \\
\text { plataforma. } \\
\text { - Foros } \\
\text { - Chats } \\
\text { - Videoconferencias entre } \\
\text { participantes }\end{array}$ & $\begin{array}{l}\text { - Autoevaluación } \\
\text { - e-test }\end{array}$ \\
\hline $\begin{array}{c}\text { xMOOC } \\
\text { Modelo conductista } \\
\text { basado en } \\
\text { contenido. }\end{array}$ & $\begin{array}{l}\text { - Videos instruccionales } \\
\text { de corta duración } \\
\text { - Lecturas } \\
\text { complementarias } \\
\text { - Materiales de apoyo } \\
\text { sugerido } \\
\text { - Recursos educativos } \\
\text { abiertos o diseñados }\end{array}$ & $\begin{array}{l}\text { - Principalmente dentro } \\
\text { de la plataforma } \\
\text { - Foros } \\
\text { - Chats } \\
\text { - Sesiones de video con el } \\
\text { autor } \\
\text { - Redes sociales como } \\
\text { apoyo }\end{array}$ & $\begin{array}{l}\text { - e-test } \\
\text { - evaluaciones peer } \\
\text { to peer }\end{array}$ \\
\hline $\begin{array}{l}\text { bMOOC } \\
\text { Modelo cognitivista } \\
\text { enfoque hibrido } \\
\text { basado en la } \\
\text { interacción humana }\end{array}$ & $\begin{array}{l}\text { - Videos instruccionales y } \\
\text { lecturas } \\
\text { - Asignaciones sobre el } \\
\text { contenido } \\
\text { - Sesiones face to face } \\
\text { con el tutor }\end{array}$ & $\begin{array}{l}\text { - Principalmente dentro } \\
\text { de la plataforma } \\
\text { - Discusiones sincrónicos } \\
\text { - Sesiones on-line }\end{array}$ & $\begin{array}{l}\text { - e-test } \\
\text { - evaluaciones peer } \\
\text { to peer } \\
\text { - trabajo grupales }\end{array}$ \\
\hline $\begin{array}{l}\text { sMOOC } \\
\text { Modelo } \\
\text { comportamentalista }\end{array}$ & $\begin{array}{l}\text { - Videos instructivos } \\
\text { semanales y lecturas } \\
\text { - Lectura } \\
\text { complementarias } \\
\text { - Recursos educativos } \\
\text { abiertos o diseñados }\end{array}$ & $\begin{array}{l}\text { - Foros } \\
\text { - Chats } \\
\text { - Sesiones de video con el } \\
\text { tutor } \\
\text { - Redes sociales como } \\
\text { apoyo }\end{array}$ & $\begin{array}{l}\text { - } \text { e-test } \\
\text { - evaluaciones peer } \\
\text { to peer }\end{array}$ \\
\hline
\end{tabular}

Tabla 1. Tipos de MOOC.

Fuente: Creador por los autores. 2018

Según Garrido, C. et al. (2015), la interacción entre estudiantes en entornos mediados por TIC, con atención a la dimensión social y afectiva ya ha sido tomada en cuenta en entornos online clásicos (Chiecher, 2013). De igual manera, la exploración de las perspectivas de las experiencias 
de aprendizaje en un MOOC, principalmente en los cursos de corte conectivista, es un factor relevante en la investigación.

Así, Mackness, Mak y Williams (2010) exploran estas perspectivas en relación con las características del conectivismo esbozadas por Downes (2009): autonomía, diversidad, apertura, y conectividad/interactividad. Por su parte, Kop y Fournier (2011) se centran en las experiencias de aprendizaje, y en particular en el aprendizaje autodirigido en el marco de un MOOC constructivista. Desde otro punto de vista, Bouchard (2009) utiliza un modelo

\section{Resultados}

Pedagógicamente la utilización de los MOOCs nos permite muchas posibilidades tecnológicas y de recursos humanos, esto ayuda de forma positiva el desarrollo de metodologías docentes innovadoras. Unas de las principales utilidades nos ofrecen los cursos MOOCS, según Navalpotro, J. A. S. (2014) son:

La capacidad de realizar encuestas para conocer los diferentes perfiles de los alumnos interesados en la realización del curso, lo que suele emplearse para mejorar sustancialmente la experiencia educativa (en nuestro caso en el ámbito de la Educación Ambiental, en general, y de la geografía en particular).

La ventaja de poder subir a la plataforma las clases en formato video acompañado de subtítulos además de la parte teórica por escrito, todo ello en formatos descargables lo que facilita que los alumnos puedan seguir el curso a su ritmo desde cualquier procedencia geográfica. Junto a esto se une la capacidad de establecer debates en vivo entre el educador y sus estudiantes mediante "chats" y video en "streaming".

La generación en el marco del propio curso de proyectos de aprendizaje (learning by doing), donde el alumno puede realizar experiencias prácticas sobre casos concretos, así como aplicar dichos conocimientos a entornos prácticos diferentes.

La monitorización a tiempo real de gran número de metadatos que permite conocer de manera científica la consecución de los objetivos del curso. Además de generar un gran dinamismo 
debido a que el docente tiene información de los resultados de manera cuantitativa semana a semana, al mismo tiempo que puede identificar los intereses de la mayoría de sus alumnos de manera cualitativa a través de las preguntas, reflexiones y debates que inserten a través de los diferentes foros de discusión que se establezcan.

El poder implantar pruebas semanales objetivas tipo test que permiten una revisión continúa de los contenidos, facilitando el aprendizaje a largo plazo.

La posibilidad de trabajos en forma de ensayos junto a su posterior evaluación por pares por parte de los compañeros del curso, lo que da lugar a la generación de mucha información formativa de gran utilidad para el alumno, debido a que ofrece una imagen real de cómo sus compañeros perciben su trabajo. Además de alcanzar un mayor grado de compromiso por parte del estudiante ya que se somete a evaluación mientras el mismo evalúa, lo que presumiblemente mejora la calidad de las votaciones.

Generar aprendizaje cooperativo a través de colectivos humanos que originen retroalimentación positiva mediante la creación de grupos temáticos por parte de los alumnos, que dé lugar a verdaderas comunidades de aprendizaje.

La capacidad técnica que tienen estos cursos de crear portafolios digitales dando lugar a la captura de información de manera inmediata, lo que establece una mejor comprensión del perfil de los estudiantes ayudando a mejorar los contenidos de los cursos ofertados.

\section{La buena elección de una plataforma confiable para los MOOC}

Son muchas las ventajas que por las que podemos considerar en la utilización una plataforma en el momento en la que deseamos desarrollar y ofertar un curso MOOC. Según Sánchez-Vera, M. (2015), una de ventajas que nos brindan es la visibilidad que ésta ofrece. Sánchez-Vera, indicia que las Universidad de Edimburgo (2013) y la Universidad de Londres (2013) han reconocido estas ventajas como uno de los factores que permite unir a una plataforma muy conocida y esta es COURSERA, se considera de elemental importancia el soporte técnico que se brindan para el buen desarrollo del curso. 
En este caso se toma como referencia esta plataforma conocidas en el medio COURSERA, ya que es muy importante estar seguros de la garantía que nos oferta al momento de querer lanzar un MOOC para la masiva participación.

Además, debemos considerad que todos los recursos que se utilizaran en el desarrollo del curso los que pueden ser audiovisuales, cuestionarios, pdf entre otros, pasen por una revisión control de calidad, esto permitirá reflejar en los participantes la calidad de los materiales y recursos se ofrece del curso.

Sánchez-Vera, M. (2015), el educador debe ceñirse a dicha clasificación, lo cual podría diferir de su intención pedagógica. Lo mismo se aplica a la evaluación, ya que como se mencionaba anteriormente, tiene unas pautas muy marcadas: se puede escoger entre preguntas tipo test en las cuales el aprendiz tiene tres intentos, y un sistema de evaluación por pares recientemente instalado cuyo protocolo solo controla la plataforma. Por tanto, si no se comulga con los principios pedagógicos propuestos por la plataforma, el diseño de un curso podría requerir altas dosis de creatividad y flexibilidad.

\section{Plataformas que ofertan cursos MOOC de forma flexible y gratuita}

\begin{tabular}{|c|c|c|}
\hline Nombre & Descripción & Sitio web \\
\hline Futurelearn & $\begin{array}{c}\text { Impulsada por la Open University de } \\
\text { Reino Unido }\end{array}$ & https://www.futurelearn.com/ \\
\hline Lore & $\begin{array}{c}\text { Incentiva la curiosidad e incluye a todas las } \\
\text { disciplinas, para personas de todas las } \\
\text { nacionalidades y edades. }\end{array}$ & http://lore.com/ \\
\hline edX & $\begin{array}{l}\text { Plataforma de cursos en línea unidos } \\
\text { basada en el MIT Open Course Ware. }\end{array}$ & https://www.edx.org/ \\
\hline UniMOOC & $\begin{array}{l}\text { Plataforma basada en el Google Course- } \\
\text { Builder y se dirige a emprendedores. }\end{array}$ & $\begin{array}{l}\text { http://iei.ua.es/mooc- } \\
\text { emprendimiento/ }\end{array}$ \\
\hline Aprendo & $\begin{array}{c}\text { Plataforma de software libre para crear y } \\
\text { administrar cursos MOOC, accesible a } \\
\text { través del sitio OpenMooc }\end{array}$ & https://github.com/OpenMOOC \\
\hline Miríada X & $\begin{array}{l}\text { Creada por Universia y Telefónica, a través } \\
\text { de la cual destacadas universidades } \\
\text { iberoamericanas ofrecen cursos online } \\
\text { gratuitos en español y portugués }\end{array}$ & https://www.miriadax.net/ \\
\hline Canvas Network & $\begin{array}{c}\text { Red abierta de cursos propone cursos a } \\
\text { docentes, estudiantes y universidades, } \\
\text { basado en el lema "Aprendizaje online } \\
\text { abierto, definido por ti" }\end{array}$ & https://www.canvas.net/ \\
\hline
\end{tabular}

Tabla 2. Plataforma que ofertan MOOC.

Fuente: Creador por los autores. 2018 


\section{Perfil del usuario de los MOOC}

Como podemos definir el tipo de usuario que es apto para la participación de un curso MOOC, a lo que queremos especificar es al participante que no sea desertor de cualquier curso al que se inscriba, para esto se debe considerar que se requiere que el estudiante sea responsable y constante al momento de ser parte de esta aprendizaje colectivo, participativo y didáctico. Para poder llegar culminar el objetico del curso que es actualizar su cocimiento siendo activo a las actividades subidas a la plataforma de estudio las mismas que son foros, chats, animaciones flash, videos con sus respectivos bancos de pregunta a ser resueltos, entre otras actividades que ofrece la plataforma con el fin de poder efectuar una construcción social del conocimiento y el aprendizaje en base a la colaboración entre los usuarios.

Poy, R., \& Gonzales-Aguilar, A. (2014), muestra en su artículo que las plataformas MOOC se fundamentan mayoritariamente en un modelo pedagógico, donde los estudiantes construyen significados por sí mismos, como lo ponen de manifiesto la presencia de las variables que caracterizan este modelo teórico.

\section{La diferencian entre la educación virtual y el MOOC}

La educación virtual desarrolla en una plataforma LMS con estructura diseñada interacción directa con el profesor entorno cerrado acceso por pago de matrícula, grupo limitado apoyo directo del profesor, comunicación mediante foros de debate orientado a la evaluación y acreditación.

MOOC sigue un diseño tecnológico que facilita la diseminación de la actividad de los participantes mediante el uso de uno o varias plataformas, entorno abierto, acceso gratuito, participación masiva, apoyo de la comunidad diversidad de herramientas de comunicación, uso de las redes sociales, énfasis en el procedimiento de aprendizaje más que en la evaluación y acreditación.

\section{Semejanzas entre la educación virtual y el MOOC}

Ambos tienen objetivos, que se quiere conseguir, hacia donde se quiere llegar y consideran los mismos factores: (quién) definir el destinatario, que un comportamiento o conducta que describe aquello que se espera que el destinatario pueda hacer, (cómo) unas condiciones o exigencias que 
tienen que estar presentes en el comportamiento, (cuanto) un grado que determina el criterio de desarrollo aceptable y permite evaluarlo.

\section{Conclusiones}

La reciente aparición de MOOC's en el desarrollo del aprendizaje a nivel superior mediante plataformas, es una de los propósitos que se desea alcanzar como parte del desarrollo del mejoramiento de la educación a nivel superior, para lo cual es necesaria la socialización de estos recursos que muy poco se los ha escuchado, para luego elaborar un proyecto destinado a la implementación plataformas MOOC en las cuales puedan participar en primera instancia a modo pilotaje los estudiantes de la universidad de modo local, para luego, una vez obtenida la experiencia de un curso abierto online masivo, poder ampliar nuestros objetivos con más cursos pero esta vez con la participación estudiantes de otras universidad a nivel nacional e internacional.

Debemos considerara que los MOOC's han tenido un rápido desarrollo en líneas de la educación, por lo cual se ha visto por su crecimiento exponencial, y la vinculación que rápidamente han tenido con estos métodos universidades de prestigio mundial.

\section{Resumen Bibliográfico}

Aretio, L. G. (2015). MOOC: ¿tsunami, revolución o moda pasajera? Revista Iberoamericana De Educación a Distancia, 18(1), 9-21. Retrieved from http://search.proquest.com/docview/1649109518?accountid=131412

Bartolomé, A., \& Steffens, K. (2015). ¿Son los MOOC una alternativa de aprendizaje? /Are MOOCs promising learning environments? Comunicar, 22(44), 91-99. Retrieved from http://search.proquest.com/docview/1675861242?accountid=131412

Blanco, Á. F., Lacleta, M. L. S., Gené, O. B., \& Peñalvo, F. J. G. (2014). EDUCACIÓN EN ABIERTO: INTEGRACIÓN DE UN MOOC CON UNA ASIGNATURA ACADÉMICA. Teoría De La Educación; Educación y Cultura En La Sociedad De La Información, 15(3), 233-255. Retrieved from http://search.proquest.com/docview/1750330748?accountid=131412 
Caldera-Serrano, J., \& León-Moreno, J. (2015). MOOC (massive online open courses) como método-plataforma educativa en el ámbito universitario 1/MOOC (massive online open courses) as a platform method-learning in the university. Documentación De Las Ciencias De La Información, 38, 301-310. doi: http://dx.doi.org/10.5209/rev_DCIN.2015.v38.50821

Castaño, C., Maiz, I., \& Garay, U. (2015). Diseño, motivación y rendimiento en un curso MOOC cooperativo/Design, motivation and performance in a cooperative MOOC course. Comunicar, 22(44), 19-26. Retrieved from http://search.proquest.com/docview/1675861848?accountid=131412

Del Cura, M, (2013). Complementos para la formación en tecnología MOOC. Universidad de Alcalá. Retrieved from http:// http://www.mcurav.es/wp-content/uploads/2013/12/MOOC.pdf

Garrido, C. C., Olazabalaga, I. M., \& Ruiz, U. G. (2015). Percepción de los participantes sobre el aprendizaje en un MOOC/ students' perceptions of a university MOOC. Revista Iberoamericana De Educación a Distancia, 18(2), 197-221. Retrieved from http://search.proquest.com/docview/1701876998?accountid=131412

González, H. M., \& Avila, A. P. (2014). Los cursos en línea masivos y abiertos (MOOC) como alternativa para la educación a distancia (massive open online courses (MOOC), an alternative to distance learning). Gecontec, 2(2), 41-49. Retrieved from http://search.proquest.com/docview/1663909125?accountid=131412

Mancera, D. T., \& Saldaña, D. G. (2014). LOS MOOCS Y SU PAPEL EN LA CREACIÓN DE COMUNIDADES DE APRENDIZAJE Y PARTICIPACIÓN (THE ROLE OF MOOCS IN THE CREATION OF LEARNING AND PARTICIPATION COMMUNITIES). Revista

Iberoamericana De Educación a Distancia, 17(1), 13-34. Retrieved from http://search.proquest.com/docview/1502621197?accountid=131412

Mancera, D. T., \& Saldaña, D. G. (2014). LOS MOOCS Y SU PAPEL EN LA CREACIÓN DE COMUNIDADES DE APRENDIZAJE Y PARTICIPACIÓN (THE ROLE OF MOOCS IN THE CREATION OF LEARNING AND PARTICIPATION COMMUNITIES). Revista

Iberoamericana De Educación a Distancia, 17(1), 13-34. Retrieved from http://search.proquest.com/docview/1502621197?accountid=131412 
Navalpotro, J. A. S. (2014). Los "MOOCs", algo más que una cuestión de escala: La educación ambiental en los albores de una sociedad tecnológica. Observatorio Medioambiental, 17, 141 205. Retrieved from https://search.proquest.com/docview/1647792662 accountid=131412

Poy, R., \& Gonzales-Aguilar, A. (2014). Factores de éxito de los MOOC: Algunas consideraciones críticas. Revista Ibérica De Sistemas e Tecnologias De Informação, 105-118. Retrieved from http://search.proquest.com/docview/1515965478?accountid=131412

Sánchez-Vera, M. (2015). Desafíos en la creación, desarrollo e implementación de los MOOC: El curso de web science en la Universidad de Southampton/Challenges in the creation, development and implementation of MOOCs: Web science course at the university of southampton. $\begin{array}{llll}\text { Comunicar, } & \text { 22(44), } & \text { 37-44. } & \text { Retrieved }\end{array}$ https://search.proquest.com/docview/1683739024?accountid=131412

Vázquez-Cano, E., \& Meneses, E. L. (2015). La filosofía educativa de los MOOC y la educación universitaria/ the educational philosophy of MOOCS and university education. Revista Iberoamericana De Educación a Distancia, 18(2), 25-37. Retrieved from http://search.proquest.com/docview/1701877398?accountid=131412 